\title{
A toddler with iron deficiency anemia unresponsive to oral iron treatment
}

\author{
Laura M. Kinlin MD MPH, Susanna Talarico MD MScCH, Melanie Kirby MBBS, Patricia C. Parkin MD
}

Cite as: CMAJ 2020 April 14;192:E393-6. doi: 10.1503/cmaj.191008

A 2-year-old girl with constipation (since 1 year of age) presented with a 1-day history of headache, vomiting and fever. Her physician ordered blood tests, which led to a diagnosis of severe nutritional iron deficiency anemia. The patient's hemoglobin level was 52 (reference interval 105-135) g/L, mean corpuscular volume was 54.0 (reference interval 70.0-90.0) fL, platelet count was 744 (reference interval $150-400$ ) $\times 10^{9} / \mathrm{L}$, white blood cell count was 12.3 (reference interval 6.0-17.5) x10\% $/ \mathrm{L}$ and serum ferritin was $3 \mu \mathrm{g} / \mathrm{L}(<12 \mu \mathrm{g} / \mathrm{L}$ is widely used as diagnostic for iron deficiency; see Appendix 1, available at www.cmaj.ca/ lookup/suppl/doi:10.1503/cmaj.191008/-/DC1, for additional information). Treatment was started with a polysaccharide-iron complex product (brand name FeraMAX) at a daily dosage of $60 \mathrm{mg}$ of elemental iron, or $4.3 \mathrm{mg} / \mathrm{kg} / \mathrm{d}$ of elemental iron (weight $13.8 \mathrm{~kg}$ [75th percentile]).

The child's fever resolved after 1 day, but the headache and emesis continued intermittently for a further 4 days; symptoms were attributed to an intercurrent viral infection. Two days after starting iron treatment, she was assessed in the emergency department of our pediatric tertiary care hospital because of persistent symptoms. Repeat blood tests showed a profound microcytic anemia (hemoglobin level $56 \mathrm{~g} / \mathrm{L}$, mean corpuscular volume $54.1 \mathrm{fL}$, marked hypochromasia and microcytes with moderate pencil forms on blood smear). Five days after starting iron treatment, the child was seen in our pediatric consultation clinic, and the dosage of polysaccharide-iron complex (FeraMAX) was increased to about $5.7 \mathrm{mg} / \mathrm{kg} / \mathrm{d}$ of elemental iron.

The child did not respond to this treatment, despite appropriate dosing and duration. In moderate-to-severe iron deficiency anemia, the hemoglobin level should increase by $10 \mathrm{~g} / \mathrm{L}$ within 2 weeks of treatment initiation; reticulocytosis peaks at around 7 days but can be seen within 72 hours (Appendix 1). Almost 4 weeks after she started the polysaccharide-iron complex (FeraMAX), the child's hemoglobin level was essentially unchanged, with no evidence of reticulocyte response (hemoglobin $55 \mathrm{~g} / \mathrm{L}$, reticulocyte count $\left.31.9 \times 10^{9} / \mathrm{L}\right)$.

When evaluating the child's refractory iron deficiency anemia, we considered treatment nonadherence (e.g., iron not given or dietary changes not implemented) and an alternative diagnosis (e.g., thalassemia or gastrointestinal disease causing malabsorption) as possible explanations. However, the clinical history was highly convincing for nutritional iron deficiency anemia: the child

\section{KEY POINTS}

- Numerous oral iron preparations (marketed under a variety of brand names) are available in Canada and are sold without a prescription as natural health products.

- Health care providers should be aware of apparent differences in effectiveness between oral iron preparations when initiating iron treatment.

- In cases of nonresponse to iron treatment, the role of iron preparation should be considered, as polysaccharide-iron complex may be less clinically effective than traditionally used ferrous iron preparations.

had never consumed any meat products, drank more than $710 \mathrm{~mL}$ (24 ounces) of cow's milk per day and was reportedly a picky eater. She had no clinical signs of malabsorption. There was no family history of thalassemia, and we did not identify hemoglobinopathy via hemoglobin-variant quantification. The patient's parents reported high adherence to iron treatment and had substantially decreased the child's milk intake (reduced to 235-475 mL daily within 5 days of starting iron treatment).

Twenty-six days after iron treatment was initiated, we recommended a change in iron preparation because of nonresponse to treatment. A ferrous iron preparation (ferrous fumarate, brand name Palafer) was started; the dosage of elemental iron $(5.7 \mathrm{mg} / \mathrm{kg} / \mathrm{d})$ was not changed. The child tolerated the change in iron preparation, without substantial worsening of constipation, and responded well. One week after she started ferrous fumarate, her hemoglobin level and reticulocyte count had increased $\left(64 \mathrm{~g} / \mathrm{L}\right.$ and $164 \times 10^{9} / \mathrm{L}$, respectively). Six weeks after initiation of the new preparation, her hemoglobin had returned to normal and she was iron replete (hemoglobin $122 \mathrm{~g} / \mathrm{L}$ and serum ferritin $32.2 \mathrm{\mu g} / \mathrm{L}$; Figure 1).

\section{Discussion}

Iron deficiency in early life remains a public health problem in Canada ${ }^{1}$ and is associated with important health effects, including neurocognitive impairments in adulthood (Appendix 1). Its prevalence in Canadian children is $12 \%-64 \%$, with Indigenous communities and low-income families disproportionately affected. ${ }^{1}$ Young children aged 1 to 3 years are particularly at risk, as dietary iron intake may be inadequate to meet increased 

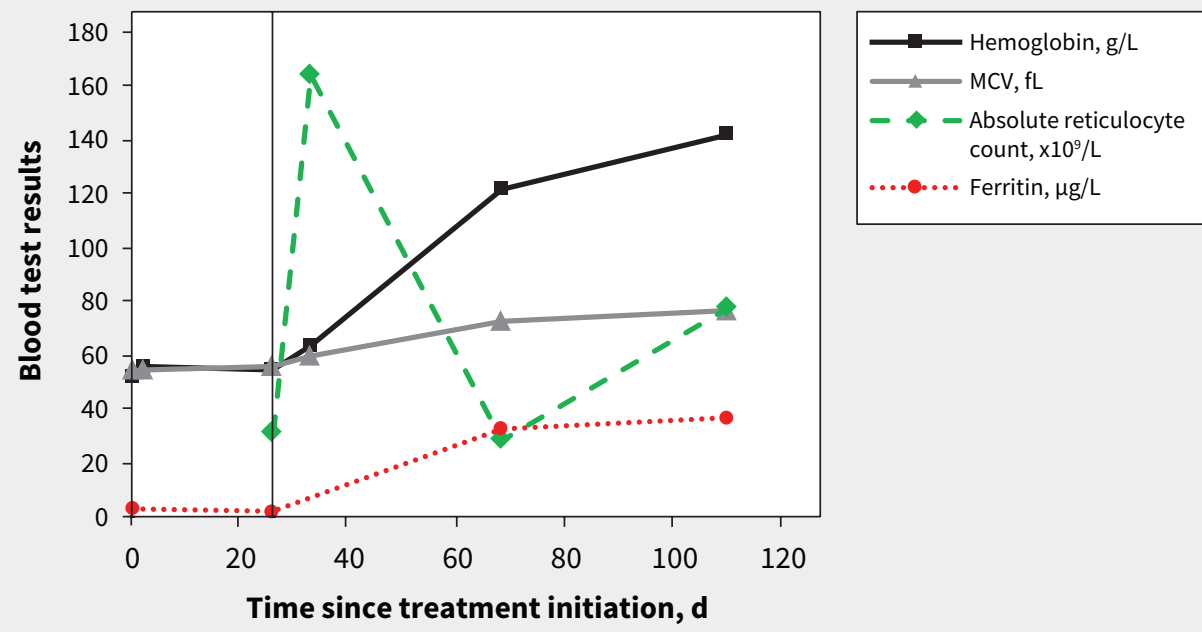

Figure 1: Serial laboratory measurements (hemoglobin, mean corpuscular volume [MCV], absolute reticulocyte count and ferritin) of a 2-year-old girl with constipation. The change from a polysaccharide-iron complex product to a ferrous fumarate product is denoted by the vertical grey line at 26 days.

physiologic requirements during a period of rapid growth. ${ }^{2}$ To prevent iron deficiency, iron-rich foods (e.g., meat; meat alternatives, such as legumes and tofu; and iron-fortified cereals) are recommended as the first complementary foods. ${ }^{3}$ Cow's milk should be introduced only after 9 to 12 months of age, and its quantity should be limited to less than $750 \mathrm{~mL}$ daily, as its iron content is low and calcium inhibits iron absorption; excess milk ingestion can also displace iron-rich foods from the diet and has been implicated in subclinical intestinal blood loss. ${ }^{3}$

\section{Mechanisms of iron absorption}

All oral iron, whether dietary or pharmacologic, is absorbed in the intestinal lumen. The precise mechanism of absorption varies according to the form of iron (Figure 2). Heme iron - found primarily in meat - is more readily absorbed, via a transport system that is not well understood. Non-heme iron - found primarily in plant-based sources and most pharmacologic iron - is less readily absorbed. Oral iron is optimally taken on an empty stomach, and separated from milk products and potentially interacting medications (Appendix 1).

\section{Management of iron deficiency anemia}

Iron deficiency is treated by addressing the cause (e.g., dietary modifications to reduce excess cow's milk) and replenishing iron stores. Elemental iron is the pharmacologically active component of oral iron preparations. In children with iron deficiency anemia, standard practice is to treat with $2-6 \mathrm{mg} / \mathrm{kg} / \mathrm{d}$ of elemental iron for 3-6 months (Appendix 1).

In Canada, many oral iron preparations are available, marketed under a variety of brand names (Appendix 2, available at www. cmaj.ca/lookup/suppl/doi:10.1503/cmaj.191008/-/DC1). Considered natural health products by Health Canada, they are generally available without a prescription and are not subject to the same regulatory process as prescription medications (Appendix 1 ).
Products vary in dose form, iron content, iron chemical state (e.g., ferrous $\left[\mathrm{Fe}^{2+}\right]$ v. ferric $\left[\mathrm{Fe}^{3+}\right]$ ) and delivery system (e.g., iron salts v. iron complexes, such as polysaccharide-iron complex).

\section{Iron salts}

Traditionally, iron deficiency has been treated with iron salts, available in either ferrous or ferric forms. Ferric salts must be reduced to the ferrous form for absorption, and the reducing capacity in the intestinal lumen may be inadequate for the amount of therapeutic ferric iron administered. Consequently, ferrous salts (i.e., ferrous sulfate, ferrous fumarate, ferrous gluconate) are preferred and are typically recommended in guidelines (Appendix 1). Their clinical effectiveness is well established; however, they are commonly associated with adverse gastrointestinal effects, likely related to unabsorbed iron affecting the intestinal mucosa (Appendix 1).

\section{Iron complexes}

Newer oral iron preparations aim for clinical effectiveness with fewer adverse effects and increased palatability. These include heme iron polypeptide (heme [bovine] iron complexed to a protein) and polysaccharide-iron complex preparations (ferric iron, mixed with a saccharide and water, released in the intestinal lumen). Heme iron polypeptide has not been formally studied in children, and its use is limited by availability only in tablet form. Polysaccharide-iron complex preparations, in contrast, are available in Canada as a water-soluble powder, easily delivered to children.

In 2017, the Canadian Agency for Drugs and Technologies in Health identified a single study examining the clinical effectiveness of polysaccharide-iron complex preparations in young children with iron deficiency ${ }^{4}$ - the BESTIRON trial, in which Powers and colleagues randomly assigned 80 children with nutritional iron deficiency anemia to ferrous sulfate or a polysaccharide-iron 


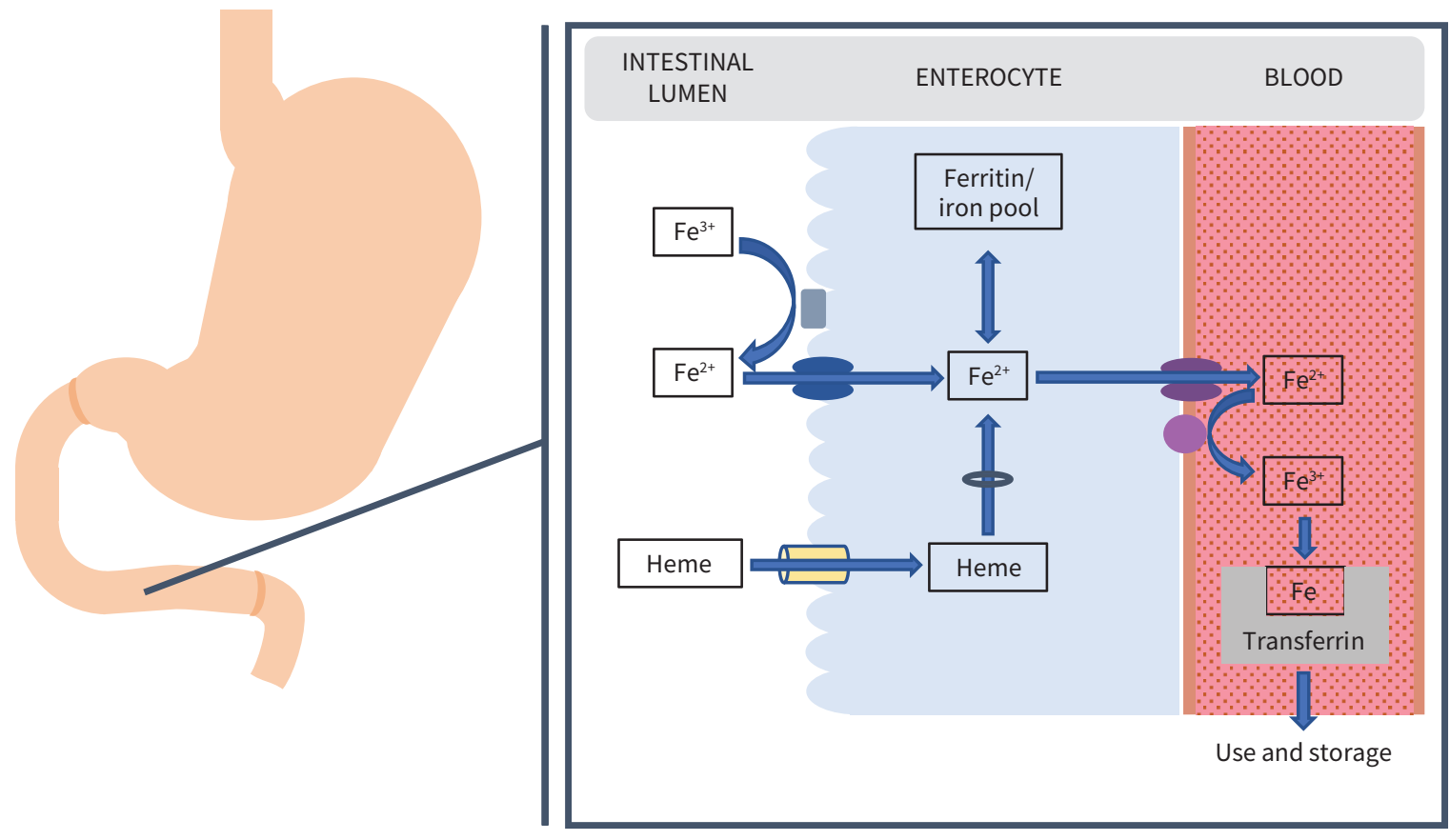

Figure 2: Simplified schematic of oral iron absorption in the duodenum. Uptake of iron from the intestinal lumen occurs at the apical surface of enterocytes. Uptake of heme iron is relatively poorly understood, but it is thought to occur via a specific heme transporter and intracellular release via a protein, heme oxygenase-1. Non-heme iron in ferric $\left(\mathrm{Fe}^{3+}\right)$ form must be reduced to the ferrous $\left(\mathrm{Fe}^{2+}\right)$ form by an enzyme in the apical brush border of the enterocyte; uptake can then occur via a divalent metal transporter. Iron can either be stored intracellularly or exported into the circulation. Transfer across the basolateral membrane of the enterocyte occurs via an iron exporter, ferroportin. Extracellular iron is then oxidized from the ferrous ( $\mathrm{Fe}^{2+}$ ) to the ferric $\left(\mathrm{Fe}^{3+}\right)$ form by an iron oxidizer (hephaestin) and binds to plasma transferrin for ultimate use or storage.

complex product at $3 \mathrm{mg} / \mathrm{kg} / \mathrm{d} .{ }^{5}$ At 12 weeks, the ferrous sulfate group had a $10 \mathrm{~g} / \mathrm{L}$ greater increase in mean hemoglobin $(p<$ $0.001)$, a greater resolution of iron deficiency anemia ( $29 \% \mathrm{v} .6 \%$, $p=0.04)$ and a greater increase in serum iron. The investigators hypothesized that polysaccharide-iron complex is less effectively absorbed than ferrous sulfate. An adult trial reported similar results, finding that a ferrous fumarate product was significantly more effective than a polysaccharide-iron complex product in increasing hemoglobin and serum ferritin levels. ${ }^{6}$

Diamond and colleagues proposed, as early as 1963 , that iron complex preparations may be less effectively absorbed than ferrous salts. ${ }^{7}$ The authors described a toddler who did not respond to 6 months of "palatable but ineffective" iron-carbohydrate complex, then rapidly improved with ferrous sulfate ${ }^{7}-$ a case similar to ours. A group of children who received treatment with iron-carbohydrate complex for 3 weeks had poor hemoglobin response and subsequently had rapid correction of anemia with ferrous sulfate. Further investigation showed a more marked increase in serum iron following ferrous sulfate administration compared with iron-carbohydrate complex. The authors concluded that poor absorption explains the latter's inadequate therapeutic effect. ${ }^{7}$

In animal studies, higher doses are required for lethal toxicity with iron complex preparations than with other oral iron preparations. ${ }^{7,8}$ Although iron accounts for a substantial proportion of deaths from accidental ingestions in children, there were no major effects or deaths in 810 potentially toxic expos- ures to polysaccharide-iron complex reported to American poison control centres. ${ }^{9}$ This reduced toxicity with polysaccharideiron complex products suggests they may be less effectively absorbed than other preparations. As Diamond and colleagues wrote in 1963, "the relative 'safety' of the iron-carbohydrate complex administered orally may represent only poor intestinal absorption." $"$

Iron-polymaltose complex - a compound similar to polysaccharide-iron complex, not available in Canada - has been more extensively studied in children. In 2 pediatric trials, iron-polymaltose complex and ferrous sulfate were equally effective. ${ }^{10,11}$ However, Bopche and colleagues documented better hemoglobin response to ferrous sulfate in another trial; hemoglobin levels decreased in 11 of 53 children receiving ironpolymaltose complex. ${ }^{12}$

\section{Conclusion}

We describe a toddler with severe nutritional iron deficiency anemia that did not respond to 1 month of a polysaccharide-iron complex product. The child's hemoglobin level and reticulocyte count rapidly responded to a ferrous iron product. Our case highlights important differences between widely available oral iron preparations: polysaccharide-iron complex preparations may be less clinically effective than traditionally used ferrous iron preparations. Health care providers should be aware of these differences, as the consequences of inadequately addressed iron deficiency in children may be substantial. 


\section{References}

1. Hartfield D. Iron deficiency is a public health problem in Canadian infants and children. Paediatr Child Health 2010;15:347-50.

2. Baker RD, Greer FR; Committee on Nutrition American Academy of Pediatrics. Diagnosis and prevention of iron deficiency and iron-deficiency anemia in infants and young children (0-3 years of age). Pediatrics 2010;126:1040-50.

3. Critch JN, Canadian Paediatric Society, Nutrition and Gastroenterology Committee. Nutrition for healthy term infants, six to 24 months: an overview. Paediatr Child Health 2014;19:547-52.

4. Wells C, Argáez C. CADTH rapid response report: summary of abstracts. Polysaccharide-iron complex for children with iron deficiency: clinical and cost-effectiveness. Ottawa: Canadian Agency For Drugs And Technologies In Health; 2017.

5. Powers JM, Buchanan GR, Adix L, et al. Effect of low-dose ferrous sulfate vs. iron polysaccharide complex on hemoglobin concentration in young children with nutritional iron-deficiency anemia: a randomized clinical trial. JAMA 2017; 317:2297-304.

6. Liu TC, Lin SF, Chang CS, et al. Comparison of a combination ferrous fumarate product and a polysaccharide iron complex as oral treatments of iron deficiency anemia: a Taiwanese study. Int J Hematol 2004;80:416-20.

7. Diamond LK, Naiman JL, Allen DM, et al. The treatment of iron-deficiency anemia - palatable but ineffective iron medication. Pediatrics 1963;31:1041-4.

8. Weaver LC, Gardier RW, Robinson VB, et al. Comparative toxicology of iron compounds. Am J Med Sci 1961;241:296-302.

9. Klein-Schwartz W. Toxicity of polysaccharide-iron complex exposures reported to poison control centers. Ann Pharmacother 2000;34:165-9.

10. Yasa B, Agaoglu L, Unuvar E. Efficacy, tolerability, and acceptability of iron hydroxide polymaltose complex versus ferrous sulfate: a randomized trial in pediatric patients with iron deficiency anemia. Int J Pediatr 2011;2011:524520.

11. Ozsurekci Y, Unal S, Cetin M, et al. Comparison of ferrous sulfate, polymaltose complex and iron-zinc in iron deficiency anemia. Minerva Pediatr 2019;71: 449-54.

12. Bopche AV, Dwivedi R, Mishra R, et al. Ferrous sulfate versus iron polymaltose complex for treatment of iron deficiency anemia in children. Indian Pediatr 2009;46:883-5.
Competing interests: Patricia Parkin received a grant from the Canadian Institutes of Health Research (CIHR) (FRN no. 115059) and nonfinancial support from Mead Johnson Nutrition (which manufactures Fer-In-Sol liquid iron) for an investigator-initiated trial of iron deficiency in young children. No other competing interests were declared.

This article has been peer reviewed.

The authors have obtained parental consent.

Affiliations: Division of Paediatric Medicine (Kinlin, Talarico, Parkin) and Haematology/Oncology (Kirby), The Hospital for Sick Children, Toronto, Ont.; Department of Paediatrics (Kinlin, Talarico, Kirby, Parkin), Faculty of Medicine, University of Toronto, Toronto, Ont.

Contributors: All authors made substantial contributions to the conception of the work, drafted the manuscript, revised the work for important intellectual content, approved the final version for publication and agreed to be accountable for all aspects of the work.

Funding: Laura Kinlin is supported by a CIHR postdoctoral fellowship (Institute of Human Development, Child and Youth Health).

Acknowledgements: The authors are indebted to the patient's family for their willingness to participate in this case report.

Correspondence to: Patricia Parkin, patricia.parkin@sickkids.ca

The section Cases presents brief case reports that convey clear, practical lessons. Preference is given to common presentations of important rare conditions, and important unusual presentations of common problems. Articles start with a case presentation (500 words maximum), and a discussion of the underlying condition follows (1000 words maximum). Visual elements (e.g., tables of the differential diagnosis, clinical features or diagnostic approach) are encouraged. Consent from patients for publication of their story is a necessity. See information for authors at www.cmaj.ca. 